\title{
Giant Emission Features at Large Distances from PNe: A Preliminary Investigation
}

\author{
C. $\operatorname{Zanin}^{1,2}$ and R. Weinberger ${ }^{1}$ \\ ${ }^{1}$ Institut für Astronomie, Universität Innsbruck, Austria; \\ ${ }^{2}$ Dipartimento di Astronomia, Universitá di Padova, Italy
}

"Senile" PNe are very old nebulae of extremely low surface brightness and large linear size; they offer e.g. the possibility of probing the conditions of the ISM also non-locally. We give three examples of huge nebulae in a preliminary inquire.

NGC 3242: An ESO Schmidt plate obtained in April 1996 shows the 'wrapped' morphology of the faint arc discovered by Deeming (1966, Astrophys. J. 146, 287) 11' south-west of this PN. Probably this arc is part of a giant halo of very low surface brightness around NGC 3242, but up to now the detection of the halo is questionable. A spectrum of the filament obtained at Las Campanas shows [O III]/H $\alpha \sim 5$ compared with a value of $\sim 3$ in the nebula (Perinotto et al. 1994, Astron. Astrophys. Supp., 107, 481). This high ratio is larger than that expected for the excitation class of NGC 3242, even larger than the maximum allowed for a photo-ionized nebula of more or less normal chemical abundance. Thus, due to the spectral characteristic and to the filamentary structure of the arc we suspect that shocks appear to play a role.

NGC 4361: A 'hole' around NGC 4361 was discovered on POSS I. Particularly noticeable is its large mean diameter of $\sim 1.5^{\circ}$ and a filament at its border, north-west of the nebula. This filament is $\sim 1.2^{\circ}$ away from the PN, which at the distance of NGC 4361 corresponds to $\sim 25 \mathrm{pc}$ ! The IRAS $100 \mu \mathrm{m}$ map as well as the $60 \mu \mathrm{m}$ map display both the filament and the hole. The IR emission points to the presence of dust. The filament could be a reflection nebula, but we cannot exclude that it is also an emission object. The position of the filament suggests that it and the hole could have been created by wind from NGC 4361.

BD +284211: On the POSS we discovered three very faint large filaments symmetrically centered on the hot $\mathrm{H}$-rich sd $\mathrm{BD}+284211$ star. Two are $\sim 2.1^{\circ}$ and $\sim 3^{\circ}$ north-west and the third is $\sim 2^{\circ}$ south-east of the star. All are elongated and curved and have a diffuse appearance without any detectable sub-structure. The BD star is, in the HR diagram, very close to the region of the CS of evolved PN (Napiwotzki, 1993, Acta Astr., 43, 343). At the distance of the BD star $(\sim 200 \mathrm{pc})$ the diameter of about $5^{\circ}$ of the whole system would correspond to the huge dimension of $17 \mathrm{pc}$.

In the near future we will be able to present a more detailed discussion of these objects as well as of newly discovered ones.

Acknowledgments: Thanks are due to the Austrian Ministry of Science, Transport and Arts, and to the FWF (project no. P10279-AST) for financial support, as well as to F. Kerber for providing a spectrum. 